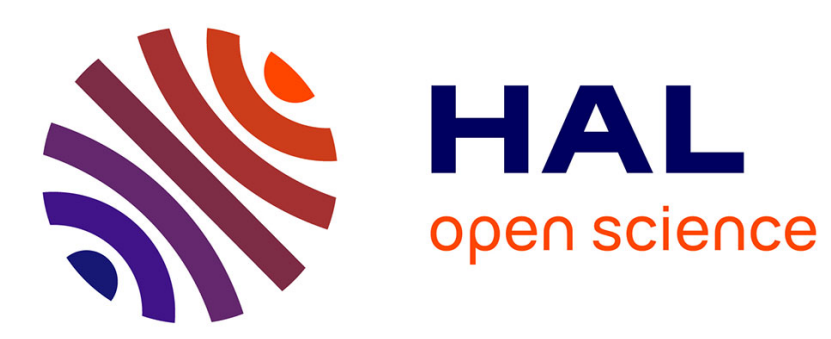

\title{
Analysis and Cancellation of Mixed-Numerologies Interference for Massive MIMO-OFDM UL
}

\author{
Xinying Cheng, Rafik Zayani, Hmaied Shaiek, Daniel Roviras
}

\section{To cite this version:}

Xinying Cheng, Rafik Zayani, Hmaied Shaiek, Daniel Roviras. Analysis and Cancellation of MixedNumerologies Interference for Massive MIMO-OFDM UL. IEEE Wireless Communications Letters, 2020, 9 (4), pp.470-474. 10.1109/LWC.2019.2959526 . hal-03082323v2

\section{HAL Id: hal-03082323 \\ https://hal.science/hal-03082323v2}

Submitted on 3 May 2021

HAL is a multi-disciplinary open access archive for the deposit and dissemination of scientific research documents, whether they are published or not. The documents may come from teaching and research institutions in France or abroad, or from public or private research centers.
L'archive ouverte pluridisciplinaire HAL, est destinée au dépôt et à la diffusion de documents scientifiques de niveau recherche, publiés ou non, émanant des établissements d'enseignement et de recherche français ou étrangers, des laboratoires publics ou privés. 


\title{
Analysis and Cancellation of Mixed-Numerologies Interference for Massive MIMO-OFDM UL
}

\author{
Xinying Cheng, Student Member, IEEE, Rafik Zayani, Member, IEEE, Hmaied Shaiek, Member, IEEE, and Daniel \\ Roviras, Senior Member, IEEE
}

\begin{abstract}
The extremely diverse service requirements is an important challenge for the future generations of wireless communication technologies. As promising solutions, orthogonal frequency division multiplexing (OFDM)-based massive multiple-input multipleoutput (MIMO) and mixed numerologies transmission are proposed. This letter investigates the transmission of different users, sharing the same bandwidth while they are associated to different numerologies. A transceiver design which aims to manage the mixed numerology spectrum sharing (SS) transmission is first presented. Then, we analyze the inter-numerology interference (INI) and derive its corresponding theoretical expressions. Based on the derived closed-form expressions, a new INI cancellation scheme is introduced, which efficiently mitigates the INI and enhances the performance of massive MIMO-OFDM uplink systems.
\end{abstract}

\section{Index Terms}

Inter-numerology interference (INI), Massive MIMO-OFDM, Mixed numerology (MN), Spectrum sharing (SS)

\section{INTRODUCTION}

$\mathbf{T}$ HE future wireless communication systems are expected to support a wide range of services with diverse requirements, where massive MIMO technology is recently recognized as a key feature. The concept was presented in [?], where the base station (BS), equipped with a large number of antennas, serves simultaneously several single-antenna users. The number of users are much smaller than the number of antennas in the BS so that spectral and energy efficiency is improved [?].

The mixed-numerologies transmission is another solution which brings more flexibility in the communication system [?], where the numerology varies based on the requirement of the service characteristics. Different numerologies refer to different parameters setting in OFDM such as subcarrier spacing (SCS), symbol duration and cyclic prefix (CP) length, which are studied in many papers [?][?]. While the implementation of numerology multiplexing significantly improves the system flexibility, the interference between users belonging to different numerologies appears and the INI affects the system performance.

A mixed-numerologies INI model is built, in [?], for single-input single-output (SISO) Windowed-OFDM systems, in which the two users occupy two adjacent subbands. Some works, such as [?][?], proposed the mixed-numerologies SS transmission but they are mainly done in a SISO system on downlink. To the best of our knowledge, general analysis and analytical expressions of the INI in massive MIMO-OFDM systems are still not presented yet. Besides, the efficient INI cancellation algorithm are needed to achieve good performance. Regarding the previous works and motivations behind this work, the main contributions of this letter are:

- A new transceiver scheme is introduced for massive MIMO-OFDM uplink system to enable flexible management of mixed numerology SS transmission.

- An analytical INI model is built for massive MIMO-OFDM system with mixed numerologies. The theoretical INI expressions are derived in closed-form to analyze theoretically the impact of INI.

- An INI cancellation method is investigated, based on the derived theoretical expressions of INI between different numerologies.

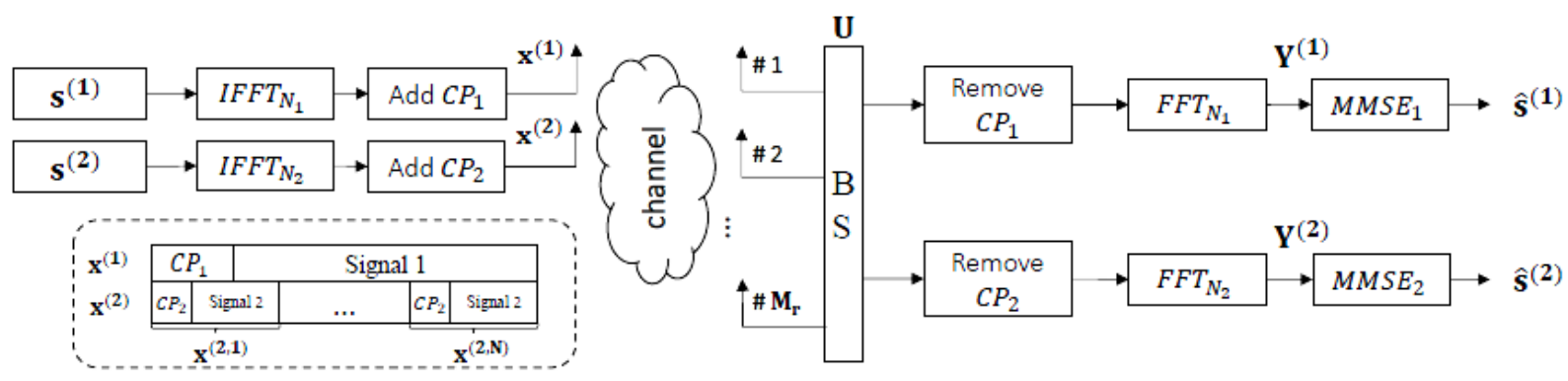

Fig. 1: System model of the massive MIMO OFDM uplink with two different numerologies: $M_{r}$ receiving antennas at the BS, two sigle-antenna terminals, two blocks illustrate two different numerologies with OFDM of $N_{1}$ and $N_{2}$ subcarriers. 
The rest of this letter is structured as follows. Section II presents the system model investigated in this study. Section III analyses the INI between different numerologies and gives the theoretical INI expressions and the patterns which influence the INI. Section IV introduces the INI cancellation based on the previous analysis. Simulation results are provided in section V. Finally, the conclusion is given in section VI.

Notations: Lower-case letters (e.g. $x$ ) , bold lower-case letters (e.g. x) and bold upper-case letters (e.g. X) stand for scalars, vectors and matrices, respectively. $\overline{\mathbf{H}}$ denotes a $M \times N \times K$ 3-dimension matrix. We denote the transpose and conjugate transpose by $\mathbf{X}^{T}$ and $\mathbf{X}^{H}$, respectively. The $k$-th element in the vector $\mathbf{x}$ is denoted as $\mathbf{x}[k]$. For a $M \times N$ matrix $\mathbf{X}, \mathbf{X}(m,:)$ denote the $m$-th line and $\mathbf{X}(:, n)$ denote the $n$-th column. $N \times N$ identity matrix, $M \times N$ all-zeros matrix, $N \times N$ discrete Fourier transform (DFT) matrix and $N \times N$ inverse discrete Fourier transform (IDFT) matrix are denoted by $\mathbf{I}_{N}, \mathbf{0}_{M \times N}$, $\mathbf{D F T}_{N}$ and $\mathbf{I D F T}_{N}$, respectively. $\operatorname{Diag}(\mathbf{x})$ represents the matrix where the diagonal elements are vector $\mathbf{x}$ and all off-diagonal elements are zero. We use $\|\mathbf{x}\|^{2}$ and $\otimes$ to denote $l_{2}$-norm of vector $\mathbf{x}$ and the convolution, respectively. $\mathbb{E}\{$.$\} stands for the$ expectation operation.

\section{SYSTEM MODEL}

We consider, in this letter, a single-cell massive MIMO-OFDM uplink system, where $M_{t}$ single-antenna users that are using different numerologies, transmit signals to a BS equipped with $M_{r}$ antennas, over a frequency-selective channel. $M_{r}$ is significantly larger than $M_{t}$. The $M_{t}$ users can be divided into $I$ groups using $I$ numerologies, represented by index $i$, where $i=1, \ldots, I . N_{i}$ and $C P_{i}$ denote the IFFT/FFT size and CP size of group $i$, respectively. The BS receives, via the $m r$-th antenna, from the $m_{t}$-th user over channel $\sqrt{\alpha_{m_{t}}} \mathbf{h t}_{m_{r}, m_{t}}$, where $\alpha_{m_{t}}$ is the large-scale fading, $\mathbf{h t}_{m_{r}, m_{t}} \in \mathbb{C}^{1 \times D}$ is the time-domain channel impulse response between user $m_{t}$ and receiving antenna $m_{r}, m_{t}=1 \ldots M_{t}, m_{r}=1 \ldots M_{r}$ and $D$ is the number of taps.

Then, $\mathbf{h f}_{m_{r}, m_{t}}^{(i)}=F F T\left(\sqrt{\alpha_{m_{t}}} \mathbf{h t}_{m_{r}, m_{t}}, N_{i}\right)$ is the channel frequency response with FFT size $N_{i}$. For a massive MIMOOFDM system, the channel frequency response is denoted by $\overline{\mathbf{H}}^{(i)} \in \mathbb{C}^{M_{r} \times M_{t} \times N_{i}}$, where

$$
\overline{\mathbf{H}}^{(i)}\left(m_{r}, m_{t},:\right)=\mathbf{h f}_{m_{r}, m_{t}}^{(i)}
$$

In order to simplify and without loss of generality, we consider, in this letter, two users $\left(M_{t}=2\right)$ using two different numerologies as shown in Fig. 1. $\mathbf{s}^{(1)}, \mathbf{s}^{(2)} \in \mathbb{C}^{1 \times N_{1}}$ are signals generated by M-quadrature amplitude modulation (QAM) for user 1 and user 2, respectively, with power $\sigma_{s}^{2}$. Considering the generalized synchronized scenario, we assume that $N_{1}=$ $N \times N_{2}, C P_{1}=N \times C P_{2}$, where $N=2^{\mu}$ and $\mu$ is an integer [?].

The received signal at the BS, $\mathbf{U} \in \mathbb{C}^{M_{r} \times\left(N_{1}+C P_{1}+D-1\right)}$, is the superposition of the signals coming from all users. The noise power at each antenna is $\frac{\sigma_{n}^{2}}{M_{r}}$.

In order to enable flexible management, we consider two detection branches, each for one numerology, at the BS, where two MMSE detectors $\overline{\mathbf{P}}^{(1)} \in \mathbb{C}^{2 \times M_{r} \times N 1}$ and $\overline{\mathbf{P}}^{(2)} \in \mathbb{C}^{2 \times M_{r} \times N_{2}}$ are used.

The received signal is separated into two branches, then, $C P_{1} / C P_{2}$ removing and $F F T_{1} / F F T_{2}$ are implemented respectively to generate the received signal for user 1 and user 2 (i.e. $\mathbf{Y}^{(1)}$ and $\mathbf{Y}^{(2)} \in \mathbb{C}^{M_{r} \times N_{1}}$ ).

Then, the data streams from the users are detected through linear processing. For $n_{i}$-th column of received signal matrix $\mathbf{Y}^{(1)}$ and $\mathbf{Y}^{(2)}$, MMSE detections are implemented by

$$
\begin{gathered}
\mathbf{r}_{n_{1}}^{(1)}=\mathbf{P}_{n_{1}}^{(1)} \mathbf{Y}^{(1)}\left(:, n_{1}\right) \quad n_{1}=1 \ldots N_{1} \\
\mathbf{r}_{n_{2}}^{(2, n)}=\mathbf{P}_{n_{2}}^{(2)} \mathbf{Y}^{(2)}\left(:,(n-1) N+n_{2}\right) \quad n_{2}=1 \ldots N_{2}, n=1 \ldots N
\end{gathered}
$$

where $\mathbf{Y}^{(1)}\left(:, n_{1}\right) \in \mathbb{C}^{M_{r} \times 1}, \mathbf{Y}^{(2)}\left(:,(n-1) N+n_{2}\right) \in \mathbb{C}^{M_{r} \times 1}$ are the $n_{1}$-th, $\left((n-1) N+n_{2}\right)$-th column of received signal matrix $\mathbf{Y}^{(1)}, \mathbf{Y}^{(2)}$, respectively. $\mathbf{r}_{n_{1}}^{(1)}, \mathbf{r}_{n_{2}}^{(2, n)} \in \mathbb{C}^{2 \times 1} . \mathbf{P}_{n_{i}}^{(i)}=\overline{\mathbf{P}}^{(i)}\left(:,:, n_{i}\right) \in \mathbb{C}^{2 \times M_{r}}, i=1,2$ denotes the MMSE detection matrix for the $n_{i}$-th OFDM subcarrier of numerology $i$, which can be given as

$$
\mathbf{P}_{n_{i}}^{(i)}=\left(\mathbf{H}_{n_{i}}^{(i)}{ }^{H} \mathbf{H}_{n_{i}}^{(i)}+\zeta \mathbf{I}_{M_{t}}\right)^{-1} \mathbf{H}_{n_{i}}^{(i)}{ }^{H}
$$

where $\mathbf{H}_{n_{i}}^{(i)}=\overline{\mathbf{H}}^{(i)}\left(:,:, n_{i}\right) \in \mathbb{C}^{M_{r} \times 2}, i=1,2$ is the frequency-domain MIMO channel of the $n_{i}$-th OFDM subcarrier with numerology $i . \zeta=\frac{\sigma_{n}^{2}}{M_{r} \sigma_{s}^{2}}$ is chosen according to the noise level.

After the detection, the estimated signal streams are extracted from $\mathbf{r}_{n_{1}}^{(1)}$ and $\mathbf{r}_{n_{2}}^{(2, n)}$, according to the following mapping for each subcarrier

$$
\begin{aligned}
\hat{\mathbf{s}}^{(1)}\left[n_{1}\right] & =\mathbf{r}_{n_{1}}^{(1)}[1] \\
\hat{\mathbf{s}}^{(2, n)}\left[n_{2}\right] & =\mathbf{r}_{n_{2}}^{(2, n)}[2]
\end{aligned}
$$




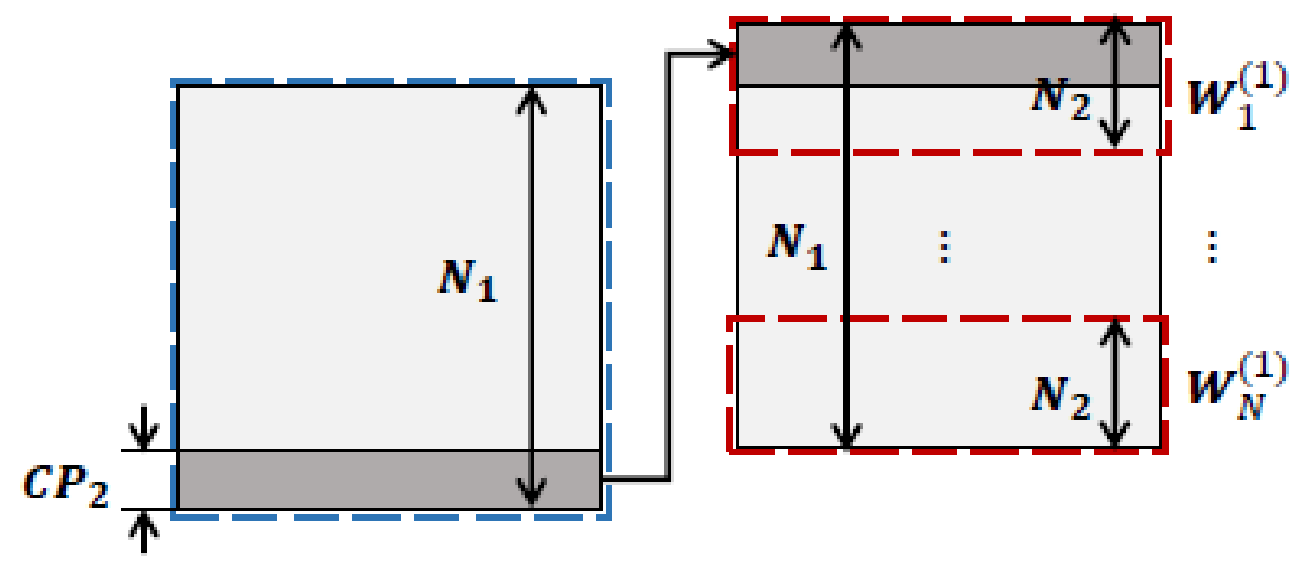

Fig. 2: Structure of matrix $\mathbf{W}^{(1)}$ and matrices $\mathbf{W}_{n}^{(1)}$.

For the analysis of INI in the following parts, we assume a noise free transmission so that the detections become zero forcing $(\mathrm{ZF})$ detection $(\zeta=0)$. In this case, the following equations are valid:

$$
\begin{gathered}
\sum_{m_{r}=1}^{M_{r}} \overline{\mathbf{P}}^{(1)}\left(2, m_{r}, n_{1}\right) \mathbf{h f}_{m_{r}, 1}^{(1)}\left[n_{1}\right] \mathbf{s}^{(1)}\left[n_{1}\right]=0 \\
\sum_{m_{r}=1}^{M_{r}} \overline{\mathbf{P}}^{(2)}\left(1, m_{r}, n_{2}\right) \mathbf{h f}_{m_{r}, 2}^{(2)}\left[n_{2}\right] \mathbf{s}^{(2, n)}\left[n_{2}\right]=0
\end{gathered}
$$

The detection matrices are designed to remove multi-user interference (MUI) within the same numerology. However, in a mixed-numerologies system, INI occurs for each user due to the usage of different numerologies in the same band. Analysis of the INI and its radiation pattern will be given in the next section.

\section{INTER-NUMEROLOGY INTERFERENCE ANALYSIS}

A. INI from numerology 1 with $N_{1}$ to numerology 2 with $N_{2}=N_{1} / N$

First, we consider the INI on user 2 with numerology 2, which uses small IFFT/FFT size. In order to only focus on the INI caused by user 1 , we consider a noise-free transmission channel and $\mathbf{s}^{(2)}=0$.

After the transmission, the signal on the $m_{r}$-th line of matrix $\mathbf{Y}^{(2)}$ can be written, in frequency domain, as

$$
\mathbf{y}_{m_{r}}^{(2, n)}=\mathbf{G}^{(1)} \mathbf{W}_{n}^{(1)} \operatorname{Diag}\left(\mathbf{h f}_{m r, 1}^{(1)}\right)\left(\mathbf{s}_{1}\right)^{T}
$$

where $\mathbf{G}^{(1)}=$ DFT $_{N_{2}} \in \mathbb{C}^{N_{2} \times N_{2}}, \mathbf{h f}_{2, m_{t}}^{(1)} \in \mathbb{C}^{1 \times N_{1}}$ is the MIMO channel frequency response between user 1 and the $m_{r}$-th receiving antenna. $\mathbf{W}_{n}^{(1)} \in \mathbb{C}^{N_{2} \times N_{1}}$ is the $n$-th $N_{2} \times N_{1}$ part of matrix $\mathbf{W}^{(1)}$, where $\mathbf{W}^{(1)} \in \mathbb{C}^{N_{1} \times N_{1}}$ is a rotated version of matrix IDFT. The structure of matrix $\mathbf{W}^{(1)}$ is shown on Fig. 2, where the blue dash block is IDFT $N_{1}$, we rotate the last $C P_{2} \times N_{1}$ part above to get $\mathbf{W}^{(1)}$ as shown on the right side. Then, $\mathbf{W}_{n}^{(1)}, n=1 \ldots N$ is part of $\mathbf{W}^{(1)}$ as the red dash blocks.

Then, the BS uses $\overline{\mathbf{P}}^{(2)}$ for the detection of user 2's signal and the interference of each subcarrier in $n$-th symbol can be expressed as

$$
\mathbf{i n i}_{n}^{(1,2)}\left[n_{2}\right]=\sum_{m_{r}=1}^{M_{r}} \overline{\mathbf{P}}^{(2)}\left(2, m_{r}, n_{2}\right) \mathbf{y}_{m_{r}}^{(2, n)}\left[n_{2}\right]
$$

From equation (10), the INI on user 2 is caused by all subcarriers of user 1.

\section{B. INI from numerology 2 with $N_{2}$ to numerology 1 with $N_{1}=N \times N_{2}$}

In this subsection, we focus on the received INI signal on user 1 with numerology 1, which uses large IFFT/FFT size. Similarly, we consider a noise-free transmission channel and $\mathbf{s}^{(1)}=0$. The signal at the $m_{r}$-th line of matrix $\mathbf{Y}^{(1)}$ can be written, in frequency domain, as 


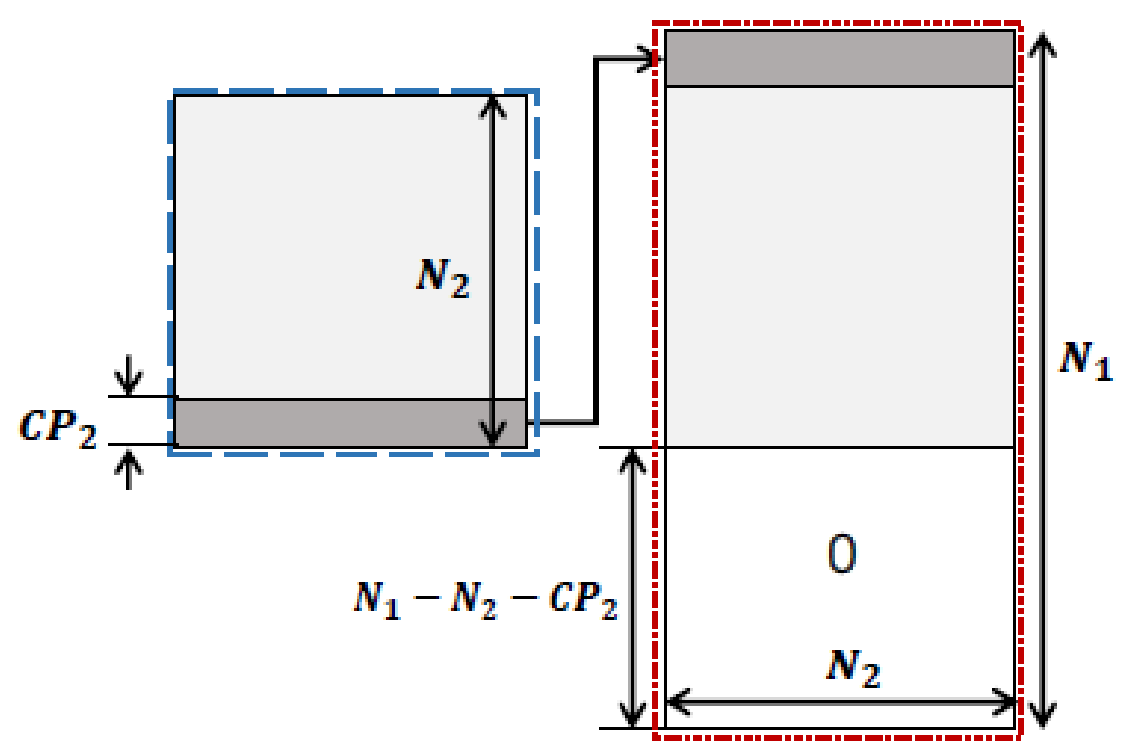

Fig. 3: Structure of matrix $\mathbf{W}_{2}^{(2)}$ with red dash box.

$$
\mathbf{y}_{m_{r}}^{(1)}=\sum_{n=1}^{N} \mathbf{E}_{n} \operatorname{Diag}\left(\mathbf{h f}_{m_{r}, 2}^{(1)}\right) \mathbf{Z}\left(\mathbf{s}^{(2, n)}\right)^{T}
$$

where $\mathbf{Z}=\mathbf{G}_{2}^{(2)} \mathbf{W}_{2}^{(2)} \in \mathbb{C}^{N_{1} \times N_{2}}$

$$
\mathbf{E}_{n} \in \mathbb{C}^{N_{1} \times N_{1}}=\left\{\begin{array}{lll}
\mathbf{G}_{1}^{(2)} \mathbf{W}_{1}^{(2)} & \text { for } & n=1 \\
\mathbf{G}_{2}^{(2)} \mathbf{I D}_{n}^{(2)} \mathbf{W}_{3}^{(2)} & \text { for } & n=2, \ldots, N
\end{array}\right.
$$

Structure of matrix $\mathbf{W}_{2}^{(2)}$ is shown on Fig. 3 with red dot block on the right side, where the blue dash block is IDFT $N_{2}$, we add the last $C P_{2} \times N_{2}$ part above and zeros below to extend the dimension to $N_{1} \times N_{2}$. All the other matrices in the expression are

$$
\begin{aligned}
& \mathbf{G}_{1}^{(2)}=\left[\begin{array}{ll}
\mathbf{0}_{N_{1} \times C P_{1}} & \mathbf{D F T}_{N_{1}}
\end{array}\right] \in \mathbb{C}^{N_{1} \times\left(N_{1}+C P_{1}\right)}, \\
& \mathbf{G}_{2}^{(2)}=\mathbf{D F T}_{N_{1}} \in \mathbb{C}^{N_{1} \times N_{1}}, \\
& \mathbf{W}_{1}^{(2)}=\left[\begin{array}{l}
\mathbf{I D F T}_{N_{1}} \\
\mathbf{0}_{C P_{1} \times N_{1}}
\end{array}\right] \in \mathbb{C}^{\left(N_{1}+C P_{1}\right) \times N_{1}}, \\
& \mathbf{W}_{3}^{(2)}=\mathbf{I D F T}_{N_{1}} \in \mathbb{C}^{N_{1} \times N_{1}}, \\
& \mathbf{I D}_{n}^{(2)}=\left[\begin{array}{ll}
\mathbf{0}_{\xi_{1} \times\left(N_{1} / 2\right)} & \mathbf{0}_{\xi_{1} \times\left(N_{1} / 2\right)} \\
\mathbf{I}_{\xi_{2}} & \mathbf{0}_{\xi_{2} \times\left(N_{1}-\xi_{2}\right)} \\
\mathbf{0}_{\xi_{3} \times\left(N_{1} / 2\right)} & \mathbf{0}_{\xi_{3} \times\left(N_{1} / 2\right)}
\end{array}\right] \in \mathbb{C}^{N_{1} \times N_{1}}
\end{aligned}
$$

for matrix $\mathbf{I D}_{n}^{(2)}$, we have

$$
\left\{\begin{array}{l}
\xi_{1}=N_{1}-(N-n+1)\left(N_{2}+C P_{2}\right) \\
\xi_{2}= \begin{cases}N_{2}+C P_{2}+D \text { for } n=2, \ldots, N-1 \\
N_{2}+C P_{2} \text { for } n=N\end{cases} \\
\xi_{3}=\left\{\begin{array}{lll}
(N-n)\left(N_{2}+C P_{2}\right)-D \quad \text { for } & n=2, \ldots, N-1 \\
(N-n)\left(N_{2}+C P_{2}\right) & \text { for } \quad n=N
\end{array}\right.
\end{array}\right.
$$

Then, the BS uses $\overline{\mathbf{P}}^{(1)}$ for the detection of user1's signal and the interference of each subcarrier can be expressed as

$$
\text { ini }^{(2,1)}\left[n_{1}\right]=\sum_{m_{r}=1}^{M_{r}} \overline{\mathbf{P}}^{(1)}\left(1, m_{r}, n_{1}\right) \mathbf{y}_{m r}^{(1)}\left[n_{1}\right]
$$

ini $^{(2,1)}\left[n_{1}\right]$ is caused by all subcarriers of user 2 .

\section{INI influencing patterns}

Corollary 1: In a constant channel, the purposed transceiver scheme for massive MIMO-OFDM based mixed numerologies SS uplink transmissions, do not generate any INI for both user 1 and user 2 (i.e. ini ${ }_{n}^{(1,2)}=0, \quad$ ini $^{(2,1)}=0$ ), due to the 
channel frequency response characteristics.

Proof: For a constant channel (i.e. the number of channel taps $D=1$ ), different size of FFTs return to the same result. For any $m t=1 \ldots M_{t}$ and $m r=1 \ldots M_{r}$, We have

for all $n_{1}=1, \ldots, N_{1}, n_{2}=1 \ldots N_{2}$.

$$
\mathbf{h f}_{m r, m t}^{(1)}\left[n_{1}\right]=\mathbf{h f}_{m r, m t}^{(2)}\left[n_{2}\right]
$$

From (4), the MMSE detection matrices originate from the channel frequency response, which, in a constant channel,

$$
\overline{\mathbf{P}}^{(1)}\left(m_{t}, m_{r}, n_{1}\right)=\overline{\mathbf{P}}^{(2)}\left(m_{t}, m_{r}, n_{2}\right)
$$

for all $n_{1}=1 \ldots N_{1}, n_{2}=1 \ldots N_{2}$.

Then, we could rewrite equation (10) and (14) in detail, to indicate the INI on each subcarrier. The INI on each subcarrier of user 1, and user 2 are expressed, respectively, in equation (20) and (19). From (15) and (16), in a constant channel, elements in vector $\mathbf{h f}_{m_{r}, 2}^{(1)}$ and vector $\mathbf{h f}_{m_{r}, 2}^{(2)}$ are identical, which leads to $\overline{\mathbf{P}}^{(1)}\left(1, m_{r}, n_{1}\right)=\overline{\mathbf{P}}^{(2)}\left(1, m_{r}, n_{2}\right), \overline{\mathbf{P}}^{(1)}\left(2, m_{r}, n_{1}\right)=$ $\overline{\mathbf{P}}^{(2)}\left(2, m_{r}, n_{2}\right)$ for all $n_{1}=1 \ldots N_{1}, n_{2}=1 \ldots N_{2}$. With these substitution and equation (7), (8), one can easily observe that ini $_{n}^{(1,2)}=0$ and ini ${ }^{(2,1)}=0$, for $n=1 \ldots N$.

$$
\begin{gathered}
\mathbf{i n i}_{n}^{(1,2)}\left[n_{2}\right]=\sum_{k_{2}=1}^{N_{2}} \sum_{n_{1}=1}^{N_{1}} \mathbf{G}^{(1)}\left(n_{2}, k_{2}\right) \mathbf{W}_{n}^{(1)}\left(k_{2}, n_{1}\right) \underbrace{\sum_{m_{r}=1}^{M_{r}} \overline{\mathbf{P}}^{(2)}\left(2, m_{r}, n_{2}\right) \mathbf{h}_{m_{r}, 1}^{(1)}\left[n_{1}\right] \mathbf{s}^{(1)}\left[n_{1}\right]}_{\text {equals to } 0 \text { in constant channel (7) }} \\
\mathbf{i n i}^{(2,1)}\left[n_{1}\right]=\sum_{n=1}^{N} \sum_{k_{1}=1}^{N_{1}} \sum_{n_{2}=1}^{N_{2}} \mathbf{E}_{n}\left(n_{1}, k_{1}\right) \mathbf{Z}\left(k_{1}, n_{2}\right) \underbrace{\sum_{m_{r}=1}^{M_{r}} \overline{\mathbf{P}}^{(1)}\left(1, m_{r}, n_{1}\right) \mathbf{h}_{m_{r}, 2}^{(1)}\left[k_{1}\right] \mathbf{s}^{(2, n)}\left[n_{2}\right]}_{\text {equals to } 0 \text { in constant channel (8) }}
\end{gathered}
$$

\section{Corollary 2: Channel selectivity and difference between $N_{1}$ and $N_{2}$ increase the INIs for both user 1 and user 2}

Proof: When the channel is frequency-selective, for all $m_{t}=1 \ldots M_{t}$ and $m_{r}=1 \ldots M_{r}$, vector $\mathbf{h f}_{m_{r}, m_{t}}^{(1)} \in \mathbb{C}^{1 \times N_{1}}$ is the interpolation of vector $\mathbf{h f}_{m_{r}, m_{t}}^{(2)} \in \mathbb{C}^{1 \times N_{2}}$, where $N-1$ values are added between every two points in $\mathbf{h f}_{m_{r}, m_{t}}^{(2)}$ to construct vector $\mathbf{h f}_{m_{t}, m_{t}}^{(1)}$. From this characteristic, we have

where $n_{1}=1, \ldots N_{1}, n_{2}=1, \ldots N_{2}$.

$$
\mathbf{h f}_{m_{r}, m_{t}}^{(1)}\left[\left(n_{2}-1\right) N+1\right]=\mathbf{h f}_{m_{r}, m_{t}}^{(2)}\left[n_{2}\right]
$$

Equation (21) indicates that, for the same time-domain channel between user $m_{t}$ and receiving antenna $m_{r}$, there are limited same-values inside the two channel frequency responses. For other values (i.e. $n_{1} \neq\left(n_{2}-1\right) N+1$ ), the difference between $\mathbf{h} \mathbf{f}_{m_{r}, m_{t}}^{(1)}\left[n_{1}\right]$ and $\mathbf{h f}_{1, m_{r}}^{(2)}\left[n_{2}\right]$ has a close relationship with the channel selectivity and the difference between $N_{1}$ and $N_{2}$. Greater selectivity and greater difference between $N_{1}$ and $N_{2}$ cause greater difference on the two channel frequency responses, which leads to greater interference.

Corollary 3: Power allocation for different users has a direct influence on the INIs at user 1 and user 2.

Proof: For the sake of simplicity and to focus on the INI analysis, in this letter, we adopt a simple power allocation scheme, where the transmitting power of the $m_{t}$-th user are equal. As noted before, the received signal matrix $\mathbf{U}$, is the summation of all the transmitted signals. Thus, the unequal receiving power for each user influences the power of INIs it generates. For example, greater large-scale fading in user $m_{t}$ (represented by $\alpha_{m_{t}}$ ) leads to smaller receiving power and smaller INI power on other users. The most remote user in the cell suffer the greatest INI.

In our system, having two users with different path-loss $\left(\alpha_{1}\right.$ and $\left.\alpha_{2}\right)$, the generated INI power from user 1 on user $2\left(p^{(1,2)}\right)$ and that from user 2 on user $1\left(p^{(2,1)}\right)$ satisfy

$$
\frac{p^{(1,2)}}{p^{(2,1)}}=\frac{\alpha_{1}}{\alpha_{2}}
$$

For example, if $\alpha_{2}<\alpha_{1}$, which indicates that user 2 is farther away from the BS than user 1 . Then, the received INI power on user 2 at the BS is higher than the INI received on user 1. On the contrary, if $\alpha_{1}<\alpha_{2}$, user 1 suffers greater INI than user 2.

\section{INI CANCELLATION}

As analysed in the previous sections, INIs are generated for each user in a frequency-selective channel. In this section, we introduce an INI cancellation scheme, with the aid of the analytical results of INI presented before. One of the main idea, in massive MIMO uplink transmission, is to reduce the complexity of the transmitters (users) while maintaining the good 
transmission quality, by doing all processing at the BS [?]. Also, in massive MIMO, the full channel state is estimated on uplink and can be used in MMSE detection and INI cancellation. In this regard, the proposed INI cancellation method is implemented at the BS side and does not add any complexity to the users. The main method is to calculate the INIs from the estimated signal $\hat{\mathbf{s}}^{(1)}$ and $\hat{\mathbf{s}}^{(2, n)}$.

Then, the signals after INI cancellation are

$$
\begin{aligned}
\tilde{\mathbf{s}}^{(1)} & =\hat{\mathbf{s}}^{(1)}-\mathbf{i n i}^{(2,1)} \\
\tilde{\mathbf{s}}^{(2, n)} & =\hat{\mathbf{s}}^{(2, n)}-\mathbf{i n i}_{n}^{(1,2)}
\end{aligned}
$$

What is more is that, the proposed INI cancellation scheme can be adapted to any system using more than two numerologies. For a given numerology, the INI cancellation can be efficiently applied by calculating and removing the INIs from all the other users, who use different numerologies. However, it is worth noticing that the proposed INI cancellation method is as sensitive to the imperfect channel state information (CSI) as the MMSE detections.

\section{SIMULATION RESULTS}

In this section, we evaluate the accuracy of the derived analytical expressions for INI in a mixed numerologies SS massive MIMO-OFDM uplink system. The BS is equipped with $M_{r}=100$ antennas and two single-antenna users $\left(M_{t}=2\right)$ that are using two different numerologies, but sharing the same band. A 16-QAM with Gray mapping is considered. Two pairs of numerologies $\left(N_{1}=1024, N_{2}=512, C P_{1}=72, C P_{2}=36\right.$ and $\left.N_{1}=1024, N_{2}=256, C P_{1}=72, C P_{2}=18\right)$ and four different channel models $(D=1,2,8,18)$ are considered, where the time-domain channel responses $\mathbf{h t}_{m r, m t}[d]=c_{d}$ have i.i.d. circularly symmetric Gaussian distributed values with zero mean and unit variance. It has to be noted that in all cases, the CP length are sufficient to suppress the inter-carrier interference (ICI) and inter-symbol interference (ISI) induced by the channel.

To evaluate the INI, we first consider the transmission without noise. We define the normalized mean-square error (NMSE) as $N M S E_{i}=\frac{\left\|\hat{\mathbf{s}}^{(i)}-\mathbf{s}^{(i)}\right\|^{2}}{\left\|\mathbf{s}^{(i)}\right\|^{2}}, i=1,2$.

The NMSE values in $\mathrm{dB}$, under different conditions for the user of interest with the transmission system introduced in this letter, are shown on TABLE I. Note that all simulation results shown in TABLE I are with $\alpha_{1}=\alpha_{2}$. One can note on TABLE I, that, INIs occur on both user except for the constant channel. Also, these results match perfectly with the analysis done in section III.C, showing that channel selectivity and difference between $N_{1}$ and $N_{2}$ increase the INIs. For example, for the same pair of numerologies, $N_{1}=1024, N_{2}=512$, there is $9 \mathrm{~dB}$ of degradation on $N M S E_{1}$ under channel $D=8$ compared to channel $D=2$ and $13 \mathrm{~dB}$ under channel $D=18$. Meanwhile, when we compare the results under the same channel selectivity, different pairs of numerologies give different performance, where users suffer more INI when the difference between IFFT/FFT size increases. For example, over the same frequency-selective channel with $D=2$, the performance of user 1 is worse when the interference is from $N_{2}=256$ than that from $N_{2}=512$ ( $4 \mathrm{~dB}$ difference).

TABLE I: NMSE (dB) of user of interest with interfering numerology and different channels

\begin{tabular}{c|c|c|c|c}
\hline $\begin{array}{c}\text { User of } \\
\text { Interest }\end{array}$ & \multicolumn{2}{|c|}{ user1 $\left(N_{1}=1024\right)$} & $\begin{array}{c}\text { user2 }\left(N_{2}=\right. \\
512)\end{array}$ & $\begin{array}{c}\text { user2 }\left(N_{2}=\right. \\
256)\end{array}$ \\
\hline $\begin{array}{c}\text { Interfering } \\
\text { User }\end{array}$ & $\begin{array}{c}\text { user2 }\left(N_{2}=\right. \\
512)\end{array}$ & $\begin{array}{c}\text { user2( } N_{2}= \\
256)\end{array}$ & \multicolumn{2}{|c}{ user1 $\left(N_{1}=1024\right)$} \\
\hline$D=1$ & -300 & -300 & -300 & -300 \\
\hline$D=2$ & -56 & -52 & -52 & -49 \\
\hline$D=8$ & -47 & -45 & -44 & -40 \\
\hline$D=18$ & -43 & -40 & -39 & -36 \\
\hline
\end{tabular}

Looking at TABLE I, the NMSE of INI is always very small ( $-36 \mathrm{~dB}$ for the maximum value) and should have a limited impact for classical SNRs encountered in real wireless communication systems. Nevertheless, it has to be pointed out that these values are for users with same path-loss: $\alpha_{1}=\alpha_{2}$. If we consider a more realistic scenario with the long term evolution (LTE) path-loss model given by [?] $\alpha_{m_{t}, d B}=128.1+37.6 \log _{10}\left(d_{m_{t}}\right)$, where $d_{m_{t}}$ is the distance from the user $m t$ to the BS in $k m$. Then, values of $\alpha_{1}$ and $\alpha_{2}$ will change. Note that $\alpha_{m_{t}, d B}$ is the value $\alpha_{m_{t}}$ in $\mathrm{dB}$, with the relationship $\alpha_{m_{t}, d B}=10 \log _{10} \alpha_{m_{t}}$. To confirm the analysis of INI with the influence of path-loss pattern, in a scenario with a cell radius equals to $1 \mathrm{~km}$, we suppose three different pair of locations: $d_{1}=d_{2}=1, d_{1}=1, d_{2}=0.3$ and $d_{1}=1, d_{2}=0.2$, which correspond to $\alpha_{1} / \alpha_{2}=0$ $\mathrm{dB},-20 \mathrm{~dB}$ and $-26 \mathrm{~dB}$, respectively.

Fig. 4 presents the symbol error rate (SER) vs. signal-to-noise ratio (SNR) of different users with/without INI cancellation, using $N_{1}=1024, N_{2}=512, D=8$, under the three different pair of locations. The SNR is defined as SNR $=M_{t} \frac{\mathbb{E}\left\{\left\|\mathbf{x}^{(i)}\right\|^{2}\right\}}{\sigma_{n}^{2}}$.

We can observe that the performance of user 1 is significantly declined when its path-loss increases compared with user 2 . For example, when $S N R=3 \mathrm{~dB}$, user 1 can achieve $S E R \leq 10^{-5}$ when $\alpha_{1} / \alpha_{2}=0 \mathrm{~dB}$ while this $\mathrm{SER}$ is improved to $10^{-3}$ when $\alpha_{1} / \alpha_{2}=-26 \mathrm{~dB}$. 


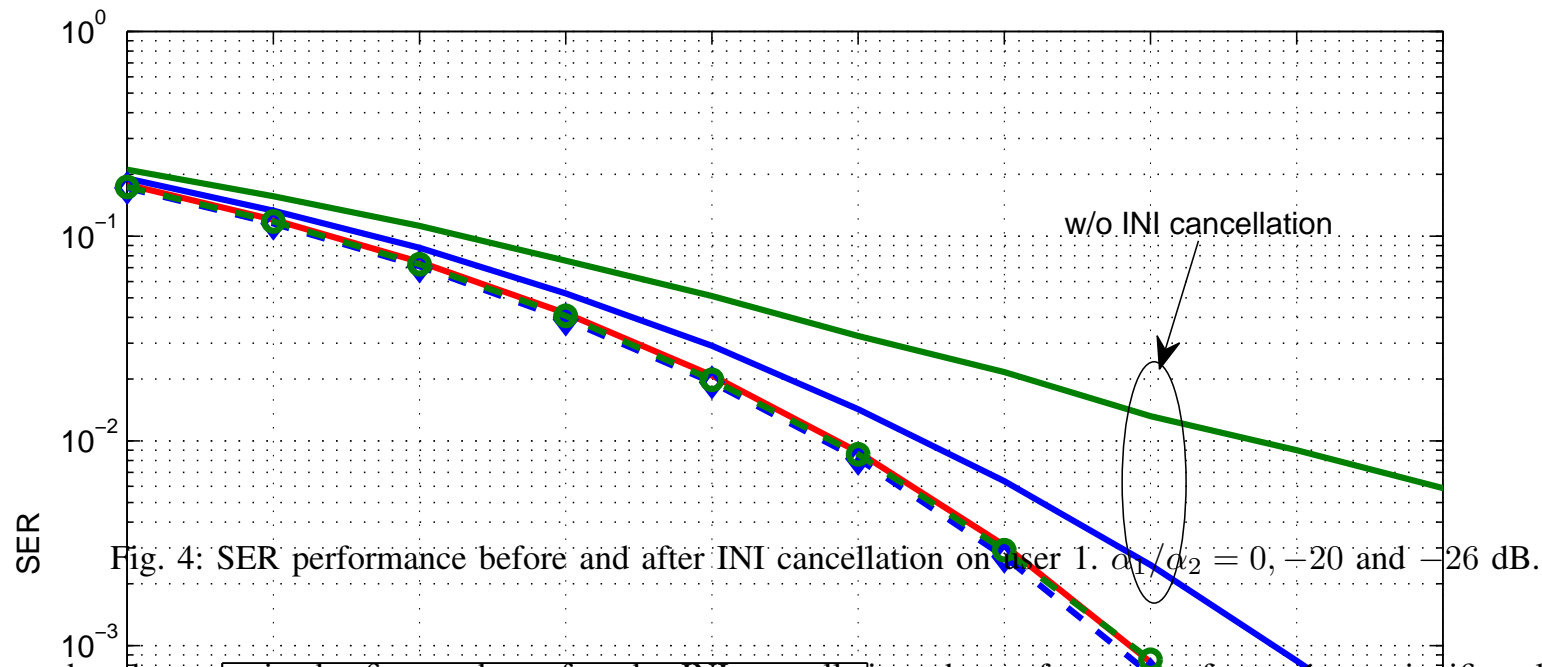

It can be fiso seen in the figurer that, affed the INI cancellation, the performan of user Xare sighificantly improved, regardless of the path-loss. These results ${ }^{2}$ can also made for user 2 in certain scenarios $\left(\alpha_{1}>\alpha_{2}>\right.$

$$
\begin{aligned}
- \text { user } 1 \alpha_{1} / \alpha_{2}=-20 \mathrm{~dB} \\
\text { user } 1 \alpha_{1} / \alpha_{2}=-26 \mathrm{~dB} \text { VI. CONCLUSION }
\end{aligned}
$$

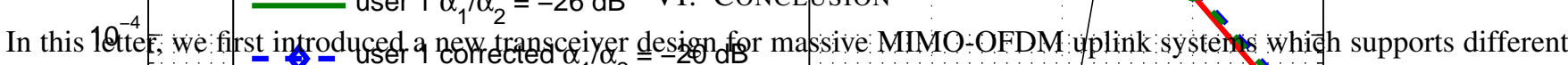
services using different numerologies while shating the same band Then we derived the theoretical axpessions of the INI,

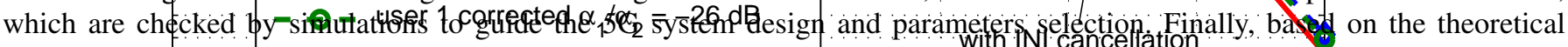
analysis, we purposed an INI cancellation. scheme. which can suppress the INI at the B.S. side without increasing the transmitter's complexity. The results show the ability of massive MIMO-OFDM system to support mixed numerologies SS transmissions

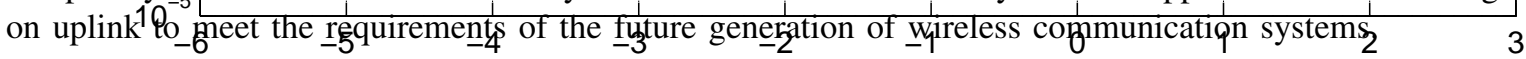

SNR dB

VII. ACKNOLEDGMENT

This work was supported in part by the framework of the ADAM5 Project receiving fund by the European Union under H2020-EU.1.3.2 with funding scheme MSCA-IF-EF-ST under Project 796401, and in part by the Scholarship of Sorbonne Université, Paris, France. 